\title{
Impact of dialysis modality on technique survival in end-stage renal disease patients
}

Jong-Hak Lee ${ }^{1,2,}$, , Sun-Hee Park ${ }^{1,2,}$, Jeong-Hoon Lim ${ }^{1,2}$, Young-Jae Park ${ }^{1,2}$, Sang Un Kim,2, Kyung-Hee Lee $e^{1,2}$, Kyung-Hoon Kim ${ }^{1,2}$, Seung Chan Park ${ }^{1,2}$, Hee-Yeon Jung ${ }^{1,2}$, Owen Kwon ${ }^{1,2}$, Ji-Young Choi ${ }^{1,2}$, Jang-Hee Cho ${ }^{1,2}$, Chan-Duck Kim ${ }^{1,2}$, and Yong-Lim Kim ${ }^{1,2}$

${ }^{1}$ Clinical Research Center for End Stage Renal Disease in Korea, ${ }^{2}$ Division of Nephrology, Department of Internal Medicine, Kyungpook National University School of Medicine, Daegu, Korea
Received: May 22, 2014

Revised : August 12, 2014

Accepted: August 19, 2014

Correspondence to

Yong-Lim Kim, M.D.

Department of Internal Med-

icine, Kyungpook National

University School of Medicine,

680 Gukchaebosang-ro, Jung-gu,

Daegu 41944, Korea

Tel: $+82-53-420-5553$

Fax: +82-53-423-7583

E-mail: ylkim@knu.ac.kr

*These authors contributed equally to this work.
Background/Aims: This study analyzed the risk factors for technique survival in dialysis patients and compared technique survival rates between hemodialysis (HD) and peritoneal dialysis (PD) in a prospective cohort of Korean patients.

Methods: A total of 1,042 patients undergoing dialysis from September 2008 to June 2011 were analyzed. The dialysis modality was defined as that used 90 days after commencing dialysis. Technique survival was compared between the two dialysis modalities, and the predictive risk factors were evaluated.

Results: The dialysis modality was an independent risk factor predictive of technique survival. PD had a higher risk for technique failure than HD (hazard ratio [HR], 10.8; 95\% confidence interval [CI], 1.9 to $62.0 ; p=0.008$ ) during a median follow-up of 11.0 months. In the PD group, a high body mass index (BMI) was an independent risk factor for technique failure (HR, 1.3; $95 \% \mathrm{CI}$, 1.0 to $1.8 ; p=0.036$ ). Peritonitis was the most common cause of PD technique failure. The difference in technique survival between $\mathrm{PD}$ and $\mathrm{HD}$ was more prominent in diabetic patients with a good nutritional status and in non-diabetic patients with a poor nutritional status.

Conclusions: In a prospective cohort of Korean patients with end-stage renal disease, PD was associated with a higher risk of technique failure than HD. Diabetic patients with a good nutritional status and non-diabetic patients with a poor nutritional status, as well as patients with a higher BMI, had an inferior technique survival rate with $\mathrm{PD}$ compared to $\mathrm{HD}$.

Keywords: Survival; Hemodialysis; Peritoneal dialysis; Body mass index; Diabetes

\section{INTRODUCTION}

The choice of dialysis modality is affected by both medical and non-medical factors, including patient preference and social and geographic factors. Although the long-term maintenance of dialysis modalities has been realized, evaluation of the risk factors associated with technique survival in dialysis is important when selecting a dialysis modality.
The long-term prognosis of end-stage renal disease (ESRD) patients according to dialysis modality remains controversial. Peritoneal dialysis (PD) is generally associated with a higher survival rate than hemodialysis (HD) in the early period after commencement of dialysis [1-3]. Moreover, PD is associated with greater preservation of residual renal function (RRF) than $\mathrm{HD}[1,4,5]$. However, it also has a considerably higher technique failure rate than HD. The various causes of technique failure in the 
early stage of $\mathrm{PD}$ (i.e., within the first year after its initiation) include infection, catheter dysfunction, peritoneal leaks, or psychosocial problems. Among these, peritonitis is the most common cause of technique failure in the early period of PD. In the later stage, the common causes of technique failure in PD include peritonitis, ultrafiltration (UF) failure, and catheter-related infection [5-7].

In Korea, several retrospective single-center studies of technique survival in PD have been reported [8-12]; however, no comparative reports on technique failure involving multicenter prospective cohorts have appeared. Therefore, to compare technique survival between dialysis modalities and to evaluate the associated factors, we analyzed technique survival in dialysis patients enrolled in the prospective observation cohort at the Clinical Research Center for End-Stage Renal Disease (CRC for ESRD) in Korea.

\section{METHODS}

\section{Patients}

A total of 32 hospitals-including 5 university-based tertiary hospitals and 26 general or provincial hospitalsparticipated in the study. From September 2008 to June 2011, a total of 1,413 incident patients were screened, of whom 342 did not meet the inclusion criteria. Thus, 1,071 incident patients were enrolled in the prospective study. After excluding 28 patients who were lost to follow-up and one patient who underwent HD and PD concordantly, a total of 1,042 incident patients were included in the study (Fig. 1). This study was performed according to the Helsinki Declaration, and the Institutional Review Board of each participating institute approved the study protocol. All of the patients provided informed consent to participate in the study.

\section{Data}

Data were collected at the initiation of enrollment and included age, sex, dialysis duration, follow-up duration, etiology of ESRD (e.g., diabetes, hypertension, glomerulonephritis), comorbidities, and smoking status. Comorbidities included chronic lung disease, peripheral vascular disease, cerebrovascular disease, congestive heart failure, arrhythmia, connective tissue disease, pep-

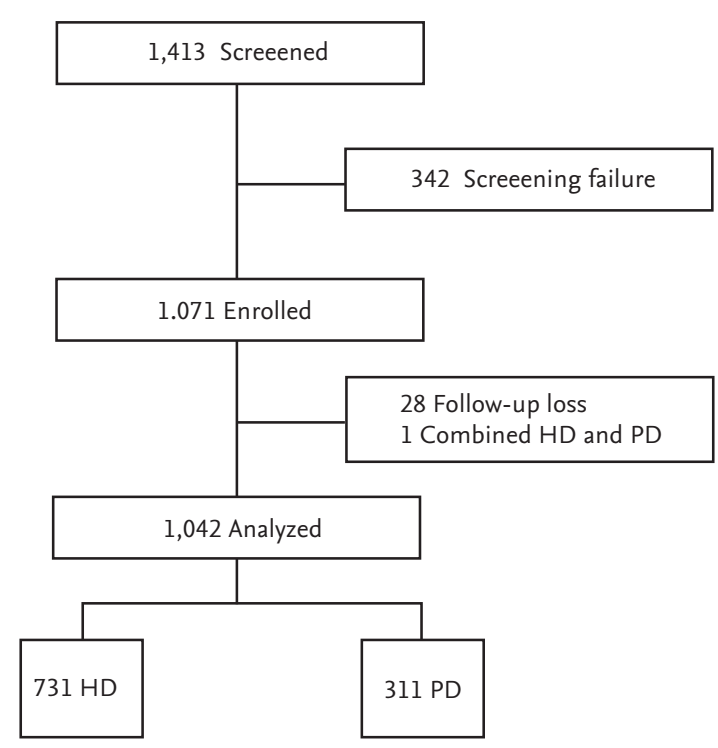

Figure 1. Patient characteristics at the Korean Clinical Research Center for end-stage renal disease from September 2008 to June 2011. HD, hemodialysis; PD, peritoneal dialysis.

tic ulcer disease, and tumor.

Laboratory data included body mass index (BMI), hemoglobin, serum albumin, and RRF, which was calculated as the average of creatinine clearance and urea nitrogen clearance from 24 hours urine collection, and was normalized according to body surface area. The nutritional status was assessed by a subjective global assessment of nutrition (SGA). In this analysis, SGA-A represents a normal nutritional status ( 6 and 7 points), SGA-B represents a mild-to-moderate malnutrition status ( 3,4 , and 5 points), and SGA-C represents a severe malnutrition status ( 1 and 2 points) [13].

\section{Definition}

The dialysis modality was defined as that in use 90 days after initiation of dialysis. Technique failure was defined as a change in dialysis modality that persisted for 6o days. Death and follow-up cessation (e.g., consent refusal, general condition exacerbation, kidney transplantation, transfer) were censored.

\section{Statistics}

The results are presented as mean \pm standard deviation or percentages. The baseline characteristics of each modality were compared by Student $t$ test for serial variation factors, and chi-square tests for independent fac- 
tors. Technique survival was analyzed by Kaplan-Meier analysis, and the log-rank test was used to evaluate differences in technique survival rate according to various factors. The independent factors associated with technique survival were extracted by multivariate Cox proportional hazards regression analysis, which was adjusted for dialysis modality, sex, age, BMI, hemoglobin, albumin, RRF, SGA, chronic lung disease, cerebrovascular disease, diabetes, congestive heart failure, and tumor. The analysis was conducted using SPSS version 19.0 (IBM Co., Armonk, NY, USA). A p value less than 0.05 was considered statistically significant.

\section{RESULTS}

\section{Baseline characteristics of the patients}

Among the 1,042 incident patients, PD patients were significantly younger than HD patients $(53.8 \pm 13.4$ years vs. $60.0 \pm 14.1$ years; $p<0.001)$. Sex distribution did not differ between patient groups (male, $60.0 \%$ vs. $60.0 \%$ in the HD and PD groups, respectively), and dialysis duration was also not different between the groups (12.8 \pm 6.9 months vs. $12.7 \pm 6.9$ months). The mean follow-up duration was $10.9 \pm 7.4$ and $11.1 \pm 7.1$ months in the HD and PD groups, respectively. Regarding the etiology of ESRD in HD and PD patients, diabetes was the most common cause $(50.2 \%$ and $46.0 \%$, respectively), followed by hypertension and glomerulonephritis. There was a significant difference in the distribution of the etiology between the groups $(p<0.001)$.

The BMI in HD patients was significantly higher than that in PD patients $(23.3 \pm 3.5$ vs. $22.7 \pm 3.4$, respectively; $p=$ o.020). Hemoglobin and serum albumin levels were not significantly different between the groups. There were also no significant differences between groups in terms of SGA and RRF. Regarding the comorbidities, chronic lung disease was significantly more common in the HD group than in the PD group ( $12.1 \%$ vs. $5.3 \%, p<0.001$ ). The tumor rate was higher in the HD group than in the PD group ( $8.6 \%$ vs. $2.6 \%, p<0.001)$. The rates of peripheral vascular disease, cerebrovascular disease, congestive heart failure, arrhythmia, connective tissue disease, and peptic ulcer disease were not significantly different between the groups. These data are summarized in Table 1.

\section{Risk factors for predicting technique survival in incident dialysis patients}

In the Cox proportional hazard analysis, the dialysis modality (hazard ratio [HR] in the PD group vs. the HD group, 10.78; 95\% confidence interval [CI], 1.87 to 62.00 ; $p=0.008)$ was the only significant factor associated with technique survival. Other factors such as sex, age, BMI, hemoglobin, serum albumin, RRF, SGA, and comorbidities did not significantly affect technique survival (Table 2).

\section{Comparison of technical survival between modali- ties}

The technique survival rate of HD was significantly higher than that of PD (Fig. 2), with 1- and 2-year technique survival rates of $99.8 \%$ and $99.0 \%$ in the HD group and $96.8 \%$ and $89.3 \%$ in the PD group, respectively. In the incident HD group, the total number of technique failures was 2. One patient requested a change to the dialysis modality from HD to PD 5 months after initiation of HD. Another patient changed to PD for control of ascites 20 months after commencement of HD. The most common cause of technique failure in PD was peritonitis (63.6\%). Other causes included inadequate dialysis (9.1\%), patient preference (9.1\%), and surgery (9.1\%) (Table 3). Among the PD patients with technique failure, 63.6\% failed to maintain PD for the first year after initiating dialysis, with $71.4 \%$ of these patients failing to maintain PD for the first 6 months.

\section{Risk factors for technique failure in incident PD patients}

We further analyzed the risk factors for PD technique failure. In incident PD patients, only a high BMI was a significant risk factor for technique failure (HR, 1.34; 95\% CI, 1.02 to $1.77 ; p=0.036)$. Moreover, the risk for technique failure increased 1.3-fold with each $1 \mathrm{~kg} / \mathrm{m}^{2}$ increase in BMI (Table 4). Sex, age, hemoglobin, albumin, RRF, SGA, and presence of comorbidities were not significant factors for technique failure in PD patients. The total patient population was divided by the median BMI value $\left(22.8 \mathrm{~kg} / \mathrm{m}^{2}\right)$. Although a significant difference in technique survival rate was found between $\mathrm{HD}$ and $\mathrm{PD}$ patients in the higher BMI group (Fig. $3 \mathrm{~A}$ ), there were no significant differences in technique survival rate in the lower BMI group (Fig. 3B). 
Table 1. Baseline characteristics of the incident dialysis patients

\begin{tabular}{|c|c|c|c|}
\hline Variable & Hemodialysis $(\mathrm{n}=731)$ & Peritoneal dialysis $(\mathrm{n}=311)$ & $p$ value \\
\hline Male sex & $439(60.0)$ & $186(60.0)$ & 0.941 \\
\hline Age, yr & $60.0 \pm 14.1$ & $53.8 \pm 13.4$ & $<0.001$ \\
\hline Dialysis duration, mon & $12.8 \pm 6.9$ & $12.7 \pm 6.9$ & 0.869 \\
\hline Follow-up duration, mon & $10.9 \pm 7.4$ & $11.1 \pm 7.1$ & 0.596 \\
\hline Etiology of ESRD & & & $<0.001$ \\
\hline Diabetes & $367(50.2)$ & $143(46.0)$ & \\
\hline Hypertension & $115(15.7)$ & $59(19.0)$ & \\
\hline Glomerulonephritis & $94(12.9)$ & $65(20.0)$ & \\
\hline Others & $155(21.2)$ & $44(14 \cdot 2)$ & \\
\hline Body mass index, $\mathrm{kg} / \mathrm{m}^{2}$ & $23 \cdot 3 \pm 3 \cdot 5$ & $22.7 \pm 3.4$ & 0.020 \\
\hline Hemoglobin, g/dL & $9.1 \pm 5.1$ & $9.1 \pm 1.5$ & 0.760 \\
\hline Serum albumin, g/dL & $3.3 \pm 0.6$ & $3.4 \pm 0.7$ & 0.099 \\
\hline Smoking, \% & & & 0.195 \\
\hline Non-smoker & $398(57.2)$ & $156(51.7)$ & \\
\hline Smoker & $82(11.8)$ & $35(11.6)$ & \\
\hline Ex-smoker & $216(31.0)$ & $111(36.7)$ & \\
\hline Subjective global assessment, \% & & & 0.372 \\
\hline A & $422(66.4)$ & $196(70.7)$ & \\
\hline $\mathrm{B}$ & $208(32.8)$ & $80(28.9)$ & \\
\hline $\mathrm{C}$ & $5(0.8)$ & $1(0.4)$ & \\
\hline $\mathrm{RRF}, \mathrm{mL} / \mathrm{min} / 1.73 \mathrm{~m}^{2}$ & $3.91 \pm 3.80$ & $4.29 \pm 3.78$ & 0.328 \\
\hline \multicolumn{4}{|l|}{ Comorbidities } \\
\hline Chronic lung disease & $85(12.1)$ & $16(5 \cdot 3)$ & $<0.001$ \\
\hline Peripheral vascular disease & $69(9.8)$ & $20(6.6)$ & 0.097 \\
\hline Cerebrovascular disease & $88(12.6)$ & $30(9.8)$ & 0.216 \\
\hline Diabetes & $419(59.0)$ & $159(51.5)$ & 0.025 \\
\hline Congestive heart failure & $101(14.4)$ & $40(13.1)$ & 0.569 \\
\hline Arrhythmia & $18(2.6)$ & $5(1.6)$ & 0.360 \\
\hline Connective tissue disease & $69(9.9)$ & $31(10.1)$ & 0.899 \\
\hline Peptic ulcer disease & $55(7 \cdot 9)$ & $21(6.9)$ & 0.583 \\
\hline Tumor & $60(8.6)$ & $8(2.6)$ & $<0.001$ \\
\hline
\end{tabular}

Values are presented as number (\%) or mean \pm standard.

ESRD, end-stage renal disease; RRF, residual renal function.

Differences in technique survival rate between dialysis modalities according to the presence of diabetes and nutritional status

We further analyzed the technique survival of each modality according to the presence of diabetes and SGA category. A statistically significant difference in technique survival rate between dialysis modalities was found in the SGA-A and diabetes groups, as well as the SGA-B, SGA-C, and non-diabetes groups (Fig. $4 \mathrm{~A}$ and $4 \mathrm{C}$ ). The SGA-A and non-diabetes groups did not show a significant difference in technique survival rate between modalities (Fig. 4B). The SGA-B, C, and diabetes groups could not be analyzed due to an insufficient number of patients in the PD group. 
Table 2. Risk of technique failure in incident dialysis patients based on the Cox model

\begin{tabular}{lccc}
\hline Variable & Reference & Hazard ratio (CI) & $p$ value \\
\hline Peritoneal dialysis & Hemodialysis & $10.78(1.87-62.00)$ & 0.008 \\
Male sex & Female & $0.89(0.21-3.88)$ & 0.879 \\
Age, yr & - & $1.03(0.97-1.09)$ & 0.301 \\
Body mass index, $\mathrm{kg} / \mathrm{m}^{2}$ & - & $1.10(0.89-1.37)$ & 0.377 \\
Hemoglobin, g/dL & - & $0.98(0.64-1.51)$ & 0.941 \\
Albumin, g/dL & - & $0.72(0.22-2.33)$ & 0.581 \\
RRF, mL/min/1.73 $\mathrm{m}^{2}$ & - & $1.03(0.84-1.26)$ & 0.798 \\
SGA 5 S & 6,7 & $4.93(0.52-47.01)$ & 0.165 \\
Chronic lung disease & No & $1.16(0.11-12.91)$ & 0.902 \\
Cerebrovascular disease & No & $1.36(0.14-12.87)$ & 0.787 \\
Diabetes & No & $1.90(0.35-10.43)$ & 0.461 \\
Congestive heart failure & No & $1.68(0.30-9.53)$ & 0.558 \\
Tumor & No & $3.68(0.31-44.40)$ & 0.306 \\
\hline
\end{tabular}

CI, confidence interval; RRF, residual renal function SGA, subjective global assessment.

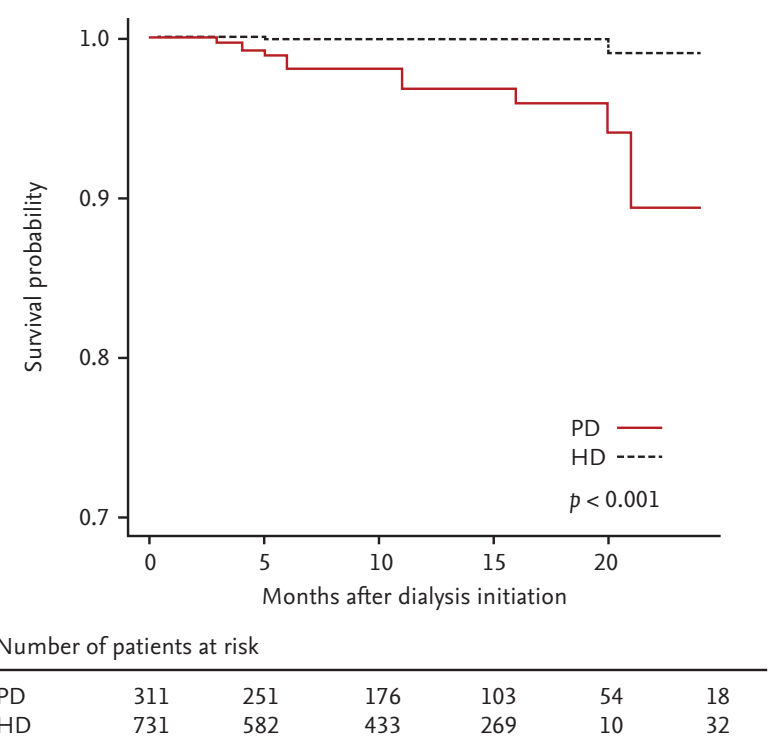

Figure 2. Technique survival rate according to dialysis modality. The technique survival rate of $\mathrm{HD}$ was higher than that of PD $(p<0.001)$. HD, hemodialysis; PD, peritoneal dialysis.

\section{DISCUSSION}

This study is the first prospective cohort study to compare technique survival according to dialysis modality in Korea. Only dialysis modality was independently associated with technique survival by multivariate analysis after adjusting for baseline characteristics. Moreover,
Table 3. Causes of technique failure in peritoneal dialysis patients

\begin{tabular}{lc}
\hline Cause of failure & Patient no. (\%) \\
\hline Peritonitis & $7(63.6)$ \\
Recurrent/persistent & $4(57.1)$ \\
\hline Acute & $3(42.9)$ \\
Inadequate dialysis & $1(9.1)$ \\
Patient preference & $1(9.1)$ \\
Surgery & $1(9.1)$ \\
Other & $1(9.1)$ \\
Total & $11(100.0)$ \\
\hline
\end{tabular}

the risk of technique failure of PD was 10-fold higher than that of HD. Similarly, previous studies in other countries reported that HD has a higher technique survival rate. In 2001, NEetherlands COooperative Study on the Adequacy of Dialysis (NECOSAD) reported a higher technique survival rate for $\mathrm{HD}$ than $\mathrm{PD}$, and the results were similar to those reported before 2000 [14]. In 2005, a cohort study from the United States reported that the rate of conversion of HD to $\mathrm{PD}$ was $<5 \%$, whereas that of conversion of PD to HD was $25 \%$ [15]. In Korea, patients with ESRD secondary to lupus nephritis showed similar results, with the technique survival rate of $\mathrm{HD}$ being higher than that of PD [16].

There are prominent characteristic differences be- 
Table 4. Risk of technique failure in incident peritoneal dialysis patients based on the Cox model

\begin{tabular}{|c|c|c|c|}
\hline Variable & Reference & Hazard ratio (CI) & $p$ value \\
\hline Male sex & Female & $1.06(0.15-7.37)$ & 0.953 \\
\hline Age, yr & - & $1.10(0.99-1.21)$ & 0.065 \\
\hline Body mass index, $\mathrm{kg} / \mathrm{m}^{2}$ & - & $1.34(1.02-1.77)$ & 0.036 \\
\hline Hemoglobin, g/dL & - & $1.25(0.56-2.82)$ & 0.589 \\
\hline Albumin, g/dL & - & $0.67(0.10-4.27)$ & 0.667 \\
\hline $\mathrm{RRF}, \mathrm{mL} / \mathrm{min} / 1.73 \mathrm{~m}^{2}$ & - & $0.84(0.60-1.17)$ & 0.306 \\
\hline $\mathrm{SGA}<5$ & 6,7 & $8.67(0.60-181.6)$ & 0.164 \\
\hline \multicolumn{4}{|l|}{ Comorbidities } \\
\hline Chronic lung disease & No & $0.25(0.00-88.85)$ & 0.640 \\
\hline Cerebrovascular disease & No & $3.33(0.16-67.29)$ & 0.434 \\
\hline Diabetes & No & $4.17(0.30-58.65)$ & 0.289 \\
\hline Congestive heart failure & No & $5.30(0.70-40.26)$ & 0.107 \\
\hline
\end{tabular}

CI, confidence interval; RRF, residual renal function; SGA, subjective global assessment.
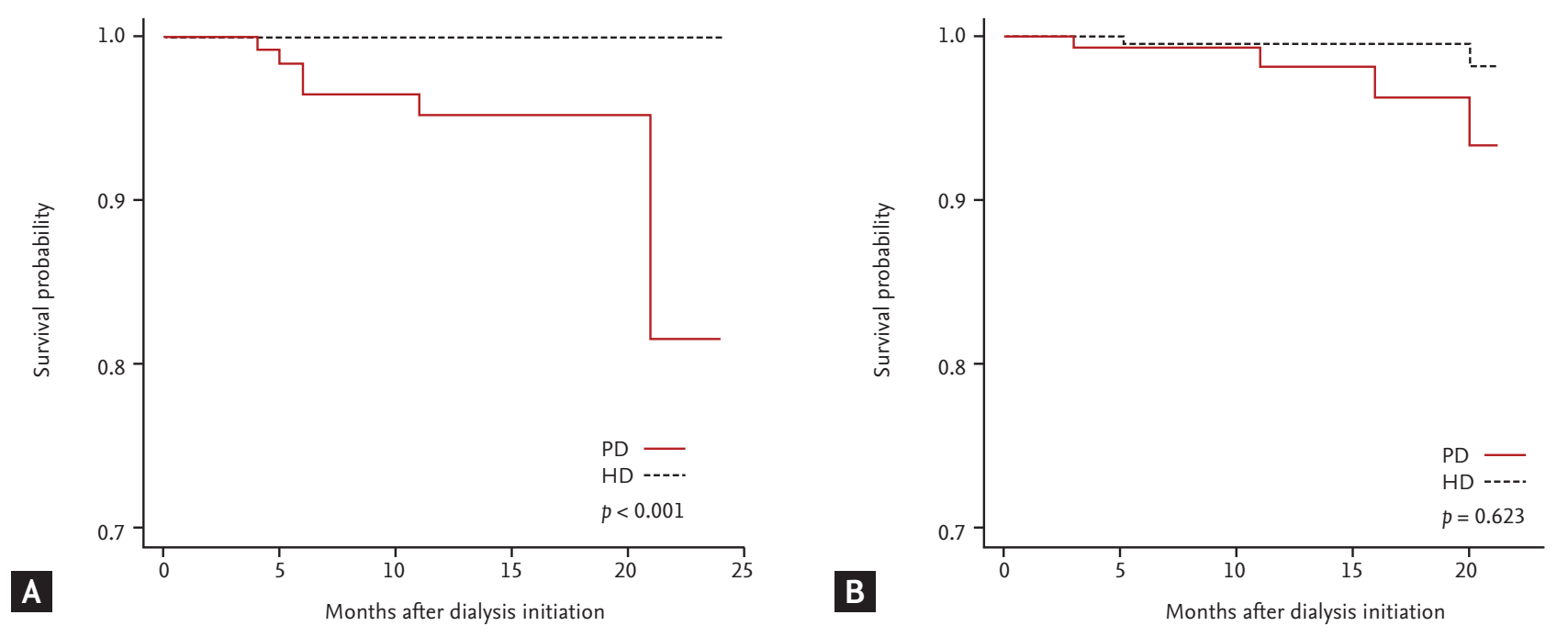

Figure 3. Technique survival rate in the higher (A) and lower (B) body mass index (BMI) groups. The higher BMI group showed a statistically significant difference in technique survival rate between dialysis modalities (A). However, the lower BMI group did not show a significant difference in technique survival rate (B). HD, hemodialysis; PD, peritoneal dialysis.

tween the two modalities, including in terms of modality access. PD catheters could be harmful in cases of severe peritonitis, so early removal may be needed depending on the presence of conditions such as fungal infection, refractory bacterial peritonitis, and encapsulating peritoneal sclerosis [17]. In contrast, vascular access for HD can be repaired repeatedly or recreated on the other side. However, HD patients who have inadequate conditions for maintaining HD such as central vein stenosis, recurrent thrombotic obstruction of vascular access, and severe congestive heart failure might be recommended to switch from HD to PD. In addition, a PD catheter can be reinserted to resume PD after resolution of severe peritoneal infection. Cases in which PD was restarted within 60 days after PD catheter removal were not counted as technique failures in our study. Although the characteristics of technique survival differed between PD and HD, both modalities could be discontinued due to various clinical conditions. Studies have compared technique survival rates between the two modalities because analysis of associated risk factors is necessary to improve the technique survival rate in di- 

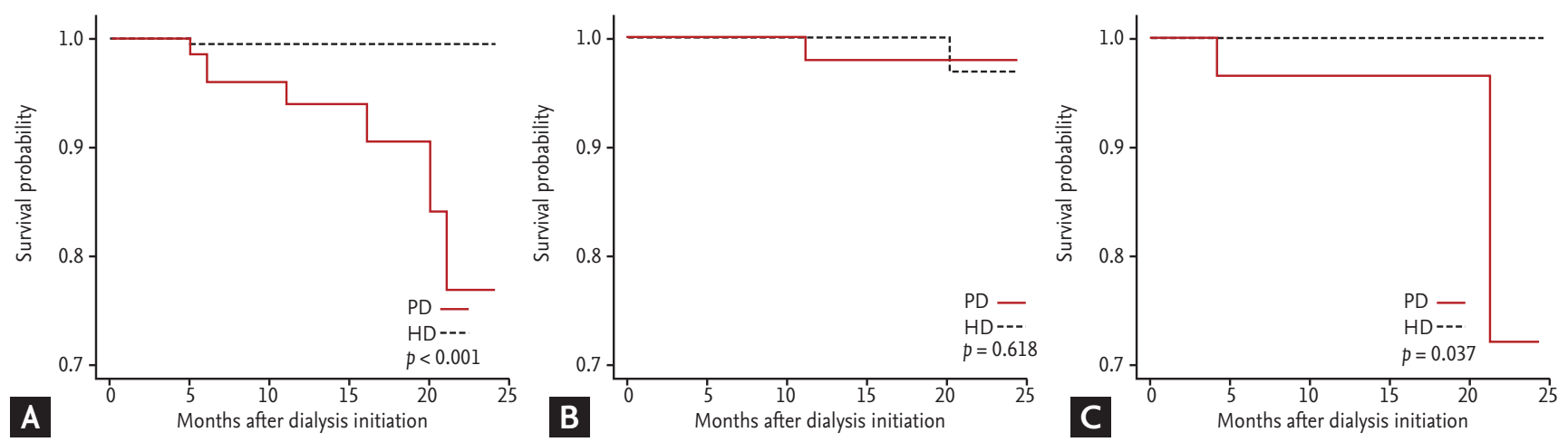

Figure 4. Technique survival rate according to the presence of diabetes and malnutrition. SGA-A \& DM (A), SGA-A \& non-DM (B), SGA-B, C, \& non-DM (C). The SGA-A and diabetes group, and SGA-B, C, and non-diabetes groups showed a statistically significant difference in technique survival rate between dialysis modalities (A, C). The SGA-A and non-diabetes groups did not show a significant difference in technique survival rate between modalities (B). SGA, subjective global assessment; DM, diabetes mellitus; HD, hemodialysis; PD, peritoneal dialysis.

alysis patients.

The causes of technique failure in PD include peritonitis, patient preference, inadequate dialysis, and surgery. Among these causes, peritonitis most commonly results in technique failure. In the present study, UF failure was not the main cause of technique failure, possibly because the study was conducted within 2 years of initiation of $\mathrm{PD}$. In a previous study from the UK, the most common cause of technique failure in PD was reported to be peritonitis, followed by poor dialysis, failed peritoneal access, wish to transfer to HD, complications of high intra-peritoneal pressure, poor UF, and cessation of dialysis [7]. Thus, our study confirms the previously reported finding that peritonitis is a leading cause of technique failure in PD. In the United States, the Choices for Healthy Outcomes in Caring for ESRD (CHOICE) study reported infections as the leading reason for transferring from PD to HD, followed by cardiovascular factors (fluid overload) and abdominal surgery, pancreatitis/malnutrition, decreased mental capacity, and abdominal wall defect [18]. On the other hand, the Australia \& New Zealand Dialysis \& Transplantation (ANZDATA) registry reported social reasons as the most common primary cause of technique failure in $\mathrm{PD}$, followed by infection, mechanical/technical complications, and dialysis failure [19]. In many Asian countries, peritonitis was the most common cause of technique failure in PD $[11,20,21]$. In Spain, peritonitis was the main reason for transfer to HD from PD, followed by UF problems, insufficient dialysis or problems related to the perito- neal catheter [22]. Thus, peritonitis remains the most common cause of technique failure in PD in most developing countries and some developed countries; however, in other countries, it has been replaced by social reasons.

BMI was the only independent risk factor for technique failure in PD in the present 2-year follow-up study. We additionally analyzed the data regarding the number of peritonitis cases in two BMI groups, dichotomized by the median BMI value. In the high BMI group, four of seven cases (57.14\%) of technique failure were attributed to peritonitis, compared to three of four patients (75.00\%) in the low BMI group. The difference was not statistically significant because of the low number of events. Therefore, whether technique failure in the high BMI group could be attributed to the higher incidence of peritonitis remains unknown. In a combined cohort study involving Chinese and Canadian centers during a 5-year follow-up that commenced in 2000, BMI, albumin, and female gender in the Chinese centers were independent predictors of technique failure in PD [21]. In Pakistan, an 18-year follow-up study that commenced in 1995 reported that BMI was the only factor significantly associated with technique survival, on the basis of a univariate analysis. However, in India, a study of BMI and PD outcomes involving four BMI groups (obese, overweight, normal, and underweight) reported that all of the BMI categories showed a similar technique survival rate with $\mathrm{PD}$, with obese patients having a greater risk of peritonitis [23]. On the basis of these results, we analyzed 
the difference in technique survival rates between dialysis modalities according to BMI category. Patients with a higher BMI had higher technique survival when HD was used as the dialysis modality.

To determine the specific factors that resulted in the difference in technique survival between dialysis modalities, we divided the two groups according to the presence of diabetes and malnutrition. The difference in technique survival between dialysis modalities was more prominent in diabetic patients with a good nutritional status and non-diabetic patients with malnutrition. These findings suggest that the technique survival rate would be higher with $\mathrm{HD}$ rather than $\mathrm{PD}$ in diabetic or malnourished patients. In general, diabetic PD patients have a higher technique failure rate [24-26]. Previous studies have suggested that poor glycemic control is associated with a higher technique failure rate in PD patients [26,27]. The effect of glycemic control using non-glucose-based PD fluid on technique survival should be investigated in future studies. The use of icodextrin-containing solutions in diabetic PD patients enhances the technique survival rate. However, this beneficial effect was attributed to improved control of body fluid status rather than good glycemic control [28]. Therefore, use of PD in diabetics should be decided on an individual-patient basis. Several reports on the relationship between nutrition and technique failure in PD patients have been published [6,10,29,30]. Serum albumin, lean body mass, and SGA had positive relationships with technique survival rate. A Chinese study reported that an increase in lean body mass reduced the HR for technique failure in PD [29]. The Canada-USA (CANUSA) study reported that a decreased serum albumin concentration was associated with an increased risk of technique failure; however, other estimates of nutrition (normalized protein catabolic rate, SGA, lean body mass) were not associated with technique failure [30]. Kwon et al. [31] reported that serum albumin had a significant effect on the technique survival rate of Korean PD patients. In this study, the nutritional status was assessed by SGA, and the results confirmed that patients with a poor nutritional status have lower technique survival rates with $\mathrm{PD}$ than in $\mathrm{HD}$.

The limitations of this study were the small number of patients with technique failure and the short follow-up period. In addition, we did not analyze the direct association between BMI and peritonitis, or between diabetic control or nutritional status and technique failure. Thus, additional long-term follow-up studies of the technique survival rates of dialysis modalities that investigate the associations between technique failure and various risk factors are warranted.

In summary, dialysis modality was the only independent risk factor predictive of technique survival in incident dialysis patients. The findings of this multicenter, prospective cohort study conducted in Korea confirms that the technique failure rate of $\mathrm{PD}$ is higher than that of $\mathrm{HD}$ during the early period of dialysis treatment. In addition, BMI was the only independent risk factor for technique failure using the early period of PD. The difference in technique failure rate between the HD and PD groups was more prominent in patients with a higher BMI and diabetic patients with a good nutritional status, as well as in non-diabetic patients with a poor nutritional status.

\section{KEY MESSAGE}

1. In a Korean prospective end-stage renal disease cohort, dialysis modality was an independent risk factor for technique failure in incident pa tients. The risk of technique failure was higher for peritoneal dialysis (PD) than hemodialysis (HD).

2. In subgroup analysis, the difference in technique survival rate between HD and PD was remarkable in patients with a higher body mass index, as well as in diabetic patients with a good nutritional status and in non-diabetic patients with a poor nutritional status.

\section{Conflict of interest}

No potential conflict of interest relevant to this article was reported.

\section{Acknowledgments}

This research was supported by a grant from the Korea Health Technology R\&D Project through the Korea Health Industry Development Institute (KHIDI), funded by the Ministry of Health and Welfare, Republic of Korea (HI15Cooor and HI10C2O2O) and supported by a National Research Foundation of Korea (NRF) grant 
funded by the Korean government (2014R1A5A2009242).

\section{REFERENCES}

1. Perl J, Bargman JM. The importance of residual kidney function for patients on dialysis: a critical review. Am J Kidney Dis 2009;53:1068-1081.

2. Wang AY, Lai KN. The importance of residual renal function in dialysis patients. Kidney Int 2006;69:1726-1732.

3. Choi JY, Jang HM, Park J, et al. Survival advantage of peritoneal dialysis relative to hemodialysis in the early period of incident dialysis patients: a nationwide prospective propensity-matched study in Korea. PLoS One 2013;8:e84257.

4. Klaric D, Knotek M. Long-term effects of peritonitis on peritoneal dialysis outcomes. Int Urol Nephrol 2013;45:519-525.

5. Sinnakirouchenan R, Holley JL. Peritoneal dialysis versus hemodialysis: risks, benefits, and access issues. Adv Chronic Kidney Dis 2011;18:428-432.

6. Lobbedez T, Boissinot L, Ficheux M, Castrale C, Ryckelynck JP. How to avoid technique failure in peritoneal dialysis patients? Contrib Nephrol 2012;178:53-57.

7. Kavanagh D, Prescott GJ, Mactier RA. Peritoneal dialysis-associated peritonitis in Scotland (1999-2002). Nephrol Dial Transplant 2004;19:2584-2591.

8. Seo HJ, Hyun SH, Kim GH, et al. Technique survival in peritoneal dialysis: a single-center experience. Korean J Med 2010;79:258-262.

9. Song YS, Jung H, Shim J, Oh C, Shin GT, Kim H. Survival analysis of Korean end-stage renal disease patients according to renal replacement therapy in a single center. J Korean Med Sci 2007;22:81-88.

10. Kim DK, Han SH, Lee JE, et al. The prognostic factors for long-term maintenance of CAPD: importance of early \%lean body mass and peritonitis. Korean J Nephrol 2007;26:590-600.

11. Han SH, Lee JE, Kim DK, et al. Long-term clinical outcomes of peritoneal dialysis patients: single center experience from Korea. Perit Dial Int 2008;28 Suppl 3:S21-S26.

12. Yoon HB, Park HC, Lee H, et al. Treatment outcomes and prognostic factors for peritoneal dialysis patients based on single center experience over 18 years. Korean J Nephrol 2009;28:19-31.

13. Steiber A, Leon JB, Secker D, et al. Multicenter study of the validity and reliability of subjective global assessment in the hemodialysis population. J Ren Nutr 2007;17:336342.

14. Jager KJ, Merkus MP, Boeschoten EW, et al. What happens to patients starting dialysis in the Netherlands? Neth J Med 2001;58:163-173.

15. Jaar BG, Coresh J, Plantinga LC, et al. Comparing the risk for death with peritoneal dialysis and hemodialysis in a national cohort of patients with chronic kidney disease. Ann Intern Med 2005;143:174-183.

16. Kang SH, Chung BH, Choi SR, et al. Comparison of clinical outcomes by different renal replacement therapy in patients with end-stage renal disease secondary to lupus nephritis. Korean J Intern Med 2011;26:60-67.

17. Li PK, Szeto CC, Piraino B, et al. Peritoneal dialysis-related infections recommendations: 2010 update. Perit Dial Int 2010;30:393-423.

18. Jaar BG, Plantinga LC, Crews DC, et al. Timing, causes, predictors and prognosis of switching from peritoneal dialysis to hemodialysis: a prospective study. BMC Nephrol 2009;10:3.

19. Brown F, Gulyani A, Dent H, Hurst K, McDonald S. Chapter 6 peritoneal dialysis [Internet]. North Terrace (AU): Australia \& New Zealand Dialysis \& Transplantation (ANZDATA), 2011 [cited 2015 Sep 20]. Available from: http://www.anzdata.org.au/anzdata/AnzdataReport/ 34thReport/2011co6_peritoneal_v1.8.pdf.

20. Unal A, Hayri Sipahioglu M, Kocyigit I, Elmali F, Tokgoz B, Oymak O. Does body mass index affect survival and technique failure in patients undergoing peritoneal dialysis? Pak J Med Sci 2014;30:41-44.

21. Fang W, Qian J, Lin A, et al. Comparison of peritoneal dialysis practice patterns and outcomes between a Canadian and a Chinese centre. Nephrol Dial Transplant 2008;23:4021-4028.

22. Remon-Rodriguez C, Quiros-Ganga P, Portoles-Perez J, et al. Results of the cooperative study of Spanish peritoneal dialysis registries: analysis of 12 years of follow-up. Nefrologia 2014;34:18-33.

23. Prasad N, Sinha A, Gupta A, et al. Effect of body mass index on outcomes of peritoneal dialysis patients in India. Perit Dial Int 2014;34:399-408.

24. Fang W, Yang X, Kothari J, et al. Patient and technique survival of diabetics on peritoneal dialysis: one-center's experience and review of the literature. Clin Nephrol 2008;69:193-200. 
25. Zimmerman SW, Oxton LL, Bidwell D, Wakeen M. Longterm outcome of diabetic patients receiving peritoneal dialysis. Perit Dial Int 1996;16:63-68.

26. Sekercioglu N, Dimitriadis C, Pipili C, et al. Glycemic control and survival in peritoneal dialysis patients with diabetes mellitus. Int Urol Nephrol 2012;44:1861-1869.

27. Tzamaloukas AH, Yuan ZY, Murata GH, Balaskas E, Avasthi PS, Oreopoulos DG. Clinical associations of glycemic control in diabetics on CAPD. Adv Perit Dial 1993;9:291294 .

28. Takatori Y, Akagi S, Sugiyama H, et al. Icodextrin increases technique survival rate in peritoneal dialysis patients with diabetic nephropathy by improving body fluid man- agement: a randomized controlled trial. Clin J Am Soc Nephrol 2011;6:1337-1344.

29. Huang JW, Lien YC, Wu HY, et al. Lean body mass predicts long-term survival in Chinese patients on peritoneal dialysis. PLoS One 2013;8:e54976.

30. Canada-USA (CANUSA) Peritoneal Dialysis Study Group. Adequacy of dialysis and nutrition in continuous peritoneal dialysis: association with clinical outcomes. J Am Soc Nephrol 1996;7:198-207.

31. Kwon HM, Kim CD, Cho JH, et al. Long term effects of dialysis adequacy, residual renal function, nutritional indices on CAPD patient outcome. Korean J Nephrol 2004;23:325-334. 